\title{
Independent Data Monitoring Committees and Heterogeneity in MRCTs: Issues and Recommendations
}

\author{
Luyan Dai ${ }^{1}$, Shuran $\mathrm{Yao}^{2^{*}}$ and Robert Makuch ${ }^{3^{*}}$ \\ ${ }^{1}$ Director, Regional Head of Statistics Asia, Biostatistics and Data Sciences Asia, Boehringer Ingelheim, Germany \\ ${ }^{2}$ Graduate Student, Regulatory Affairs Program, Yale University, USA \\ ${ }^{3}$ Professor of Biostatistics and Director of Regulatory Affairs Program, Yale University, USA
}

"Corresponding Author: Shuran Yao, 60 College Street, LEPH 203, New Haven CT USA 06520, E-mail: shuran.yao@yale.edu Robert Makuch, 60 College Street, LEPH 203, New Haven, CT USA 06520, Tel: 203-785-2842; Fax: 203-785-6912; E-mail: robert.makuch@yale.edu

Received Date: Dec 04, 2017; Accepted Date: Dec 08, 2017; Publish Date: Dec 14, 2017

Copyright: (c) 2017 Dai L, et al. This is an open-access article distributed under the terms of the Creative Commons Attribution License, which permits unrestricted use, distribution and reproduction in any medium, provided the original author and source are credited.

\section{Introduction}

With the globalization trend in drug development, Multi-regional Clinical Trials (MRCTs) have become a common practice adopted by numerous pharmaceutical firms around the world.

By design, MRCTs synchronize the drug development schedule across regions with the goal of expediting the overall process of obtaining marketing authorization globally. The primary reasons for conducting MRCTs are to make effective and safe new drugs available to patients worldwide with minimal time lags. With proper planning, MRCTs can reduce or eliminate the need of duplicated trials done sequentially over time in separate countries. Such a programmatic approach, if taken, could lead to ethical and regulatory problems because a positive outcome in one country could preclude use of the same study with the same control arm in other countries. MRCTs also help to fulfill the recruitment requirement in a restricted timeframe especially when required sample sizes are very large or the disease of interest is rare.

Much has been written about the acceptability of foreign clinical data in the context of MRCTs. For example, the International Conference on Harmonization (ICH) has numerous guidelines that describe how clinical data can be used for broad-based approval globally. In ICH E5, the stated objectives include:

1. Describe the characteristics of foreign clinical data that will facilitate their extrapolation to different populations and support their acceptance as a basis for registration of a medicine in a new region;

2. Describe regulatory strategies that minimize duplication of clinical data and facilitate acceptance of foreign clinical data in the new region;

3. Describe development strategies capable of characterizing ethnic factor influences on safety, efficacy, dosage and dose regimen.

The ICH also has published its E17 guideline that describes general principles for planning and designing MRCTs. The purpose of this guideline is to provide strategic planning initiatives as they relate specifically to MRCTs. This guideline, dedicated exclusively to MRCTs, shows the importance that these studies will have in future drug development at the global level.

A critical part of any randomized clinical trial (RCT) is that it be conducted ethically, with protection of the patient of paramount importance as part of the study's design and conduct. The standard approach to insuring ethical integrity is through an Independent Data Monitoring Committee (IDMC). Part of the duties and responsibilities of the IDMC is to not only insure the study's ethical propriety and patient protections, but also to guarantee that the study will provide scientifically valid results. Thus, the IDMC must be fully aware of the special and unique features of MRCTs as described in ICH guidelines including E3, E4, E5, E6, E8, E9, E10, and E18 [1-9]. Because of this special status accorded MRCTs, the IDMCs for such trials also must be designed with full appreciation of MRCT characteristics and possibly different regulatory requirements that are unique to MRCTs.

The purpose of this paper is to describe IDMC duties and responsibilities for MRCTs. Some less-than-familiar issues specific to MRCTs are discussed.

\section{Independent Data Monitoring Committee (IDMC) for MRCTs}

According to FDA guidelines, an IDMC is a group of individuals with pertinent expertise that reviews on a regular basis accumulating data from an ongoing clinical trial. IDMC main activities include:

1. Maintaining appropriate firewalls when communicating with the Sponsor to insure regulatory integrity;

2. Reviewing the study protocol prior to study initiation;

3. Reviewing recruitment once the study is underway;

4. Reviewing protocol dropouts, violations and deviations especially those that may affect the scientific validity of the trial;

5. Monitoring safety, reviewing efficacy, and evaluating benefit: risk, and finally;

6. Giving recommendations on continuation, modification or early termination of the trial.

\section{Ideal MRCT Settings}

In an MRCT, the IDMC is responsible for correctly evaluating the benefit: risk as quickly as possible in order to protect patients in the trial. Standard IDMC activities are sufficient for IDMC decisionmaking with ideal MRCT data. In an ideal MRCT setting, accumulating data and results are replicable among different countries, regions, demographic groups, and so on. Results among primary and secondary endpoints are consistent, supportive and cohesive. Data are of high quality, coming from a study that is well designed and conducted. Overall results are robust, statistically highly persuasive, and there is no evidence of qualitative interactions in which treatment benefit is markedly reversed among different patient subgroups in the trial. Straightforward benefit: risk evaluation can be performed readily due to the lack of qualitative interactions. 
To exemplify in an area especially pertinent to our discussions in this paper, regional effects are consistent with the overall effects. Both the point estimate and the confidence interval fall on the same side of treatment benefit across all regions and subgroups. That is, only quantitative interactions are observed between treatment and subgroups, where the new drug demonstrates superiority over the control consistently over all subgroups. As discussed in later section, qualitative interaction is more common in the complicated reality world of MRCTs. This leads to critical issues on how an IDMC should interpret such results and make its recommendations how the trail should proceed.

Figure 1 sets an example of "ideal" MRCT data. The result was taken from the Anglo-Scandinavian Cardiac Outcomes Trial-Blood Pressure Lowering Arm (ASCOT-BPLA), where patients were assigned to either Amlodipine-based group or the Atenolol-based group [10,11]. The Amlodipine-based group can be thought as the new drug treatment group in the MRCT while the Atenolol-based group is the control. The secondary and tertiary endpoints mirror the regions and subgroups in MRCTs.

With the exception of the "Fatal/nonfatal HF" row, all other factors have point estimates together with upper bounds of the $95 \%$ confidence interval to the left side of 1.0 indicating the null hypothesis of no difference between treatments. Only quantitative interaction is present. Treatment effect is statistically significant for all levels.

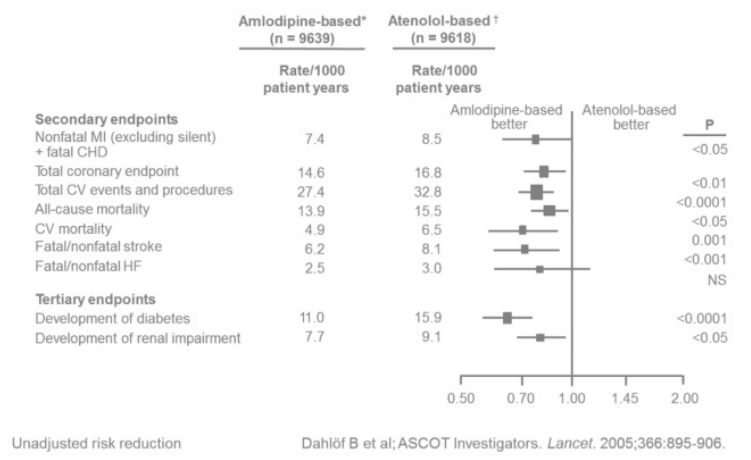

Figure 1: ASCOT-BPLA: Additional reductions in group receiving the amlodipine-based regimen.

\section{Challenges in Reality}

Ideal MRCT outcomes, exemplified above, are desirable but are many times unattainable in reality. Regional heterogeneity exists in every MRCT, and the real question is whether that heterogeneity is quantitative or qualitative.

Intrinsic factors such as genetic polymorphism, distribution of body weight or age are recognized to have potential impacts on pharmacokinetic (PK)/pharmacodynamic (PD) outcomes and therefore influencing drug safety and efficacy that contribute to the regional heterogeneity.

Extrinsic factors such as culture, medical practice, therapeutic approach, regulatory requirements and socio-economic status could impact study results during study recruitment, conduct measurement and retention approach.
In addition, the observed treatment effects and heterogeneity across regions can be possibly attributed to chance finding due to the limited region-specific patient data.

Currently, requirement and implementation guidelines of MRCTs vary across regulatory agencies. The US FDA issued multiple guidance documents regarding the acceptance of clinical data from studies conducted outside the US. The European EMA published similar guidelines. China issued an MRCT guideline in 2015. Under the leadership of Japan, the APEC Regulatory Committee developed an MRCT Roadmap.

The US sets no specific requirement on the number or percentage of US patients in MRCTs. Approval can be obtained with zero patients treated in the US. However, there is obligation to justify that demographics and standard of care in MRCTs reflect the US patient population [8]. EMA MRCT guidance asks for about 25\% of patients treated in the European Union (EU) for approval. But if an argument can be made of an equivalent/ similar therapeutic environment and patient population with the trial population, similarly to US, approvals can be obtained with zero patients treated in the EU [12]. China requires local data from Mainland Chinese subjects in general. Chinese sample size should meet statistical requirement for efficacy evaluation in the single MRCT. Safety data from multiple studies are often emphasized by the Chinese FDA (CFDA) [13]. The Pharmaceuticals and Medical Devices Agency (PMDA) guidelines proposed two methods to plan local Japanese patients for the consistency of evaluation in MRCTs. Although room exists to adjust based on recruitment feasibility and therapeutic settings, the calculation is commonly required as a reference to plan how many Japanese patients would be entered into MRCTs [14].

\section{Complicated Reality of MRCTs: Presence of Substantial Heterogeneity}

Substantial heterogeneity (SH) observed in MRCTs will trigger IDMC concerns.

Figure 2 shows the MRCT results from the Metoprolol ControlledRelease Randomized Intervention Trial in Heart Failure (MERIT-HF) [15]. The overall results strongly favor TOPROL-XL over placebo with the $95 \%$ confidence interval far beyond the relative risk $(R R)=1.0$ baseline. Yet looking at the US regional result alone, the point estimate on RR of total mortality favors placebo over TOPROL-XL, with the 95\% confidence interval expanding widely towards both sides containing the point 1 .

Assume that these data were based on a study that is still ongoing. Qualitative interaction of this type may be more likely to occur in ongoing studies since the sample size of the entire study has not been met. This type of substantial, qualitative regional heterogeneity immediately would raise ethical concerns especially on US patients assigned to the TOPROL-XL group.

To the IDMC members, intermediate trial results of this type in an ongoing study would be cause of great concern and deliberations. Questions arise on whether this observation reflects a true unfavorable effect for the US patients, or is it merely a play of chance. 


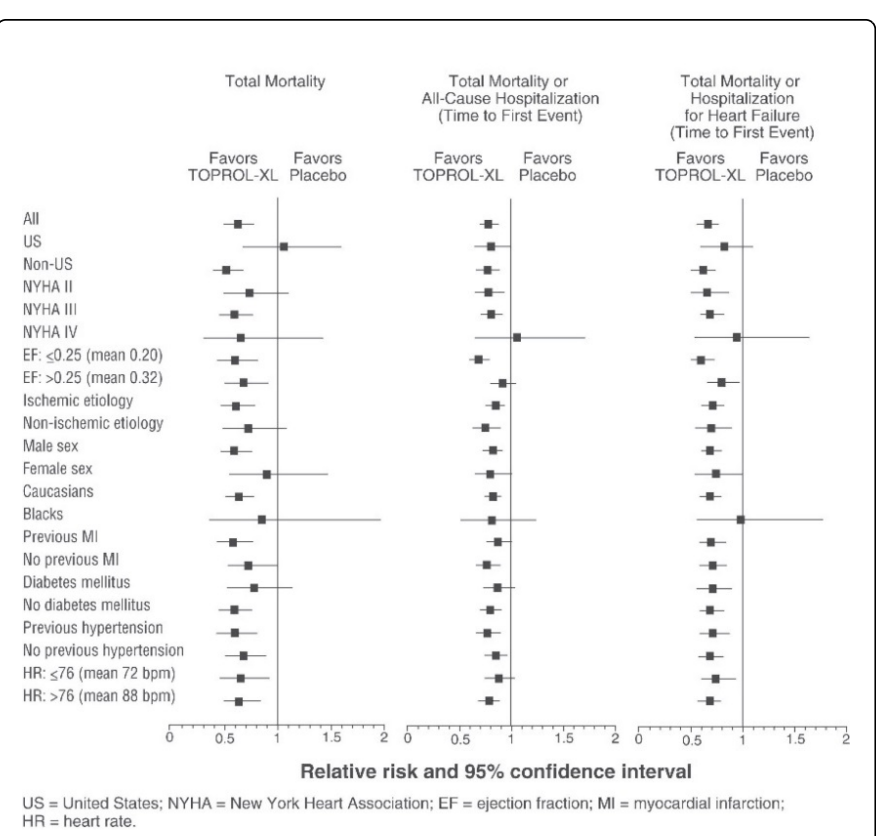

Figure 2: Results for subgroup in MERIT-HF.

Do these outcomes supports regionalized medicine preferences? What are the possible sources are to explain SH? Should the trial be stopped for the US patients only? Or should it be put on hold for the US patients until further evaluations have been done to assess reasons for harm in this region? Should the $\mathrm{SH}$ be ignored and the overall study results be the basis for determining whether the study should continue as is, be modified, stop accrual for the entire MRCT until reasons for harm in the US population are understood, or terminate the entire study prematurely? The IDMC needs to decide whether to recommend possible protocol and study conduct changes at an early stage in order to minimize $\mathrm{SH}$.

Besides these questions that the IDMC faces in its decision-making process, a few more considerations should be given in presence of $\mathrm{SH}$. While maintaining appropriate firewalls in communication with sponsors, should the IDMC contact the sponsor when such SH occurs. For the outlier regions, the IDMC may assess the potential to bring back the treatment effect estimate towards the favorable direction using statistical approaches such as Bayesian methods. The overriding IDMC issue becomes, how much weight are the overall study results given in comparison to region-specific or other subgroup-specific results? What actions should be taken? These represent thorny issues for IDMCs to consider when involved in MRCTs, and represent new areas where further ethical and statistical discussions are needed.

\section{Recommendations for MRCT IDMCs}

One face-to-face meeting of the IDMC is mandatory near the start of the study. The IDMC face-to-face meeting is best when combined with an Executive Committee or senior investigator meeting. Wider discussions are then easier to undertake and decisions to be reached.

Because of the complexity of MRCTs, the composition of the IDMC must be carefully considered to address the different types of development programs and areas of expertise required.
It is recommended to finalize the IDMC Charter at the face-to-face meeting, or very soon thereafter. IDMC activities should be broadbased and include review of data quality, study conduct, safety, efficacy, substantial heterogeneity, and risk versus benefit data. Time devoted to each of these activities should shift over time, with data quality and study conduct playing a more important role early on and followed later by increased emphasis on outcome data and $\mathrm{SH}$ examination.

The IDMC should discuss tables, listings and graphs (TLG) that will address issues including but not limited to blinding, pooling, and region-specific and subgroup-specific results.

Examination of heterogeneity must be a key agenda item, and a consensus must be reached on ways the IDMC plans to explore and deal with substantial homogeneity. IDMC should discuss beforehand how large regional and other subgroup differences can become before certain actions described in the Charter will be implemented. The nature and extent of SH may set off an alarm that requires additional IDMC actions-these needs to be clearly specified in advance in the IDMC Charter.

\section{Summary}

MRCTs are truly complex due to global involvement of regionspecific regulatory, operational, clinical, and ethical entities. Different MRCT characteristics may require a fundamentally different IDMC composition and proposed set of activities. These efforts must be addressed in the IDMC Charter. As always, a sense of community among the IDMC members is crucial to a successful decision-making process in protecting the patients.

\section{References}

1. Structure and Content of Clinical Study Reports: ICH E3 (1995) The International Conference on Harmonisation.

2. Dose-Response Information to Support Drug Registration: ICH E4 (1994) The International Conference on Harmonisation.

3. Ethnic factors in the acceptability of foreign clinical data: ICH E5 (R1) (1998) The International Conference on Harmonisation.

4. Good Clinical Practice: ICH E6 (R2) (2016) The International Conference on Harmonisation.

5. General Considerations for Clinical Trials: ICH E8 (1997) The International Conference on Harmonisation.

6. Statistical Principles for Clinical Trials: ICH E9 (1998) The International Conference on Harmonisation.

7. Choice of Control Group and Related Issues in Clinical Trials: ICH E10 (2000) The International Conference on Harmonisation.

8. General Principles for Planning and Design of Multi-Regional Clinical Trials: ICH E17 (2014) The International Conference on Harmonisation.

9. Genomic Sampling and Management of Genomic Data: ICH E18 (2017) The International Conference on Harmonisation.

10. Dahlof B, Sever PS, Poulter NR, Wedel H, Beevers DG, et al. (2005) Prevention of cardiovascular events with an antihypertensive regimen of amlodipine adding perindopril as required versus atenolol adding bendroflumethiazide as required, in the Anglo-Scandinavian Cardiac Outcomes Trial-Blood Pressure Lowering Arm (ASCOT-BPLA): a multicentre randomised controlled trial. Lancet, 366: 895-906.

11. Ethnic factors in the acceptability of foreign clinical data: ICH E5 (R1) (1998) The International Conference on Harmonisation.

12. Reflection paper on the extrapolation of results from clinical studies conducted outside the EU to the EU-population (CHMP/EWP/ 692702/2008) (2009) Committee for Medicinal Products for Human Use (CHMP). 
Citation: Dai L, Yao S, Makuch R (2017) Independent Data Monitoring Committees and Heterogeneity in MRCTs: Issues and Recommendations. Pharm Regul Aff 6: 197. doi:10.4172/2167-7689.1000197

Page 4 of 4

13. Provisions for Drug Registration (2007) China Food and Drug Administration.

14. Basic Principles on Global Clinical Trials (2007) Pharmaceuticals and Medical Devices Agency (PMDA)
15. Wedel H, Demets D, Deedwania P, Fagerberg B, Goldstein S, et al. (2001) Challenges of subgroup analyses in multinational clinical trials: experiences from the MERIT-HF trial. Am Heart J 142: 502-511. 